\title{
Models for Nonlinear Elastomers
}

\author{
H.T. Banks and Laura K. Potter \\ Center for Research in Scientific Computation \\ North Carolina State University \\ Raleigh, NC 27695-8205 USA \\ Gabriella A. Pinter \\ Department of Mathematical Sciences \\ University of Wisconsin Milwaukee \\ Milwaukee, WI 53201-0413 USA \\ M.J. Gaitens and L.C. Yanyo \\ Thomas Lord Research Center \\ Lord Corporation \\ Cary, NC 27511-7900 USA
}

\section{Introduction}

Models involving nonlinear partial differential equations become more and more widespread as theoretical and computational tools for their analysis advance. Well-known (and still partially unsolved) problems include, for example, the Euler and Navier-Stokes equations modeling the motion of a fluid (or air) which is a central problem in aircraft design. New computational tools make possible simulations, predictions, model development through inverse problems and inclusion of nonlinear effects in commercial finite element packages. In many areas new, more refined models are needed as modern applications 'outgrow' the traditional linear assumptions. In some cases the need for the accurate prediction of transient phenomena makes the inclusion of nonlinearities imperative.

An important area where nonlinear models have a potential impact is the rubber industry. Elastomeric materials can be found in diverse engineering applications e.g., in springs, bearings, shock absorbing bushes, helicopter rotor suspensions, tires, vibration suppression devices, bridge supports. They appear both as passive damping devices and actively controllable smart materials. These new applications motivate the need for a better understanding of the mechanical behavior of rubber-like composites which is a necessary first step in the design of both passive and active material devices $[11,16]$. The dynamic behavior of elastomers is very complex. They exhibit significant nonlinearities in both geometric and material characteristics. Typical nonlinear behaviors of the stress and strain in rubber materials under finite deformation include a continuous increase of strain at decreasing rates upon loading, variable magnitudes of the strain subject to rates of loading, and different loading and unloading paths due to hysteretic memory effects. In addition, the current state of the material depends on the strain/strain-rate history, the type and amount of filler in the material and the temperature. The nonlinear effects are particularly strong for large 
strains and for highly filled elastomers. Our focus in modeling is to accurately capture the nonlinear dynamic and hysteretic effects.

\section{Models for elastomeric materials}

Traditionally there are two approaches to modeling rubber materials. There are physics or molecularbased theories that try to describe the microscopic behavior of the particles and fibers that constitute the material, and there are phenomenological models that treat the material as a continuum $[12,14,15,21,22,23]$. Phenomenological models are based on the assumption that the material is isotropic, i.e., the long chain molecules are randomly oriented in the unstrained state. Loading causes orientation of the molecules, but it is in the direction of the loading so the assumption of isotropy remains valid. Most such models utilize the strain energy density to describe the state of the material. However, the proposed strain energy functions can typically capture only the current state of the rubber and cannot distinguish between different loading or unloading histories. Thus, these models cannot accurately describe the significant hysteresis exhibited by filled rubber samples.

\subsection{Model development}

In developing a model for the dynamic behavior of filled elastomers we considered two basic deformations: extension and shear. Our hysteretic models are based on the basic models developed by Banks, et.al., in [4, 6]. First we considered the model of a rubber rod under uniaxial tension. (Our shear results are briefly summarized at the end of Section 2.2.) The Timoshenko theory for longitudinal vibrations of a rubber bar with a tip mass leads to

$$
\begin{aligned}
& \rho A_{c} \frac{\partial^{2} u}{\partial t^{2}}-\left(C_{D} \frac{\partial^{2} u}{\partial t \partial x}+A_{c} \frac{\partial \sigma}{\partial x}\right)=0 \quad 0<x<\ell \\
& M \frac{\partial^{2} u}{\partial t^{2}}(t, \ell)=-\left.\left(A_{c} \sigma+C_{D} \frac{\partial^{2} u}{\partial t \partial x}\right)\right|_{x=\ell}+f(t)+M g \\
& u(t, 0)=0 \\
& u(0, x)=\varphi_{0}, \quad u_{t}(0, x)=0,
\end{aligned}
$$

where $u$ is the deformation in the $x$ direction, $\rho$ is the mass density, $f(t)$ is the applied external force, $A_{c}$ is the cross sectional area, $M$ is the tip mass, $g$ is the gravitational constant and $\sigma$ is the stress. This model includes a Kelvin-Voigt type term as a first approximation to damping. The stress-strain relationship in the basic model is

$$
\sigma(t)=G(\tilde{e}(t))
$$

where $\tilde{e}$ is the finite strain (since we are interested in large deformations) and it is given by

$$
\tilde{e}=\frac{\partial u}{\partial x}+\frac{1}{2}\left(\frac{\partial u}{\partial x}\right)^{2}=\varepsilon+\frac{1}{2} \varepsilon^{2}
$$

where $\varepsilon=\frac{\partial u}{\partial x}$ is the usual infinitesimal strain of linear elasticity. However, modeling the nonlinear behavior between the stress and the finite strains $\tilde{e}$ (which are themselves nonlinear functions of the 


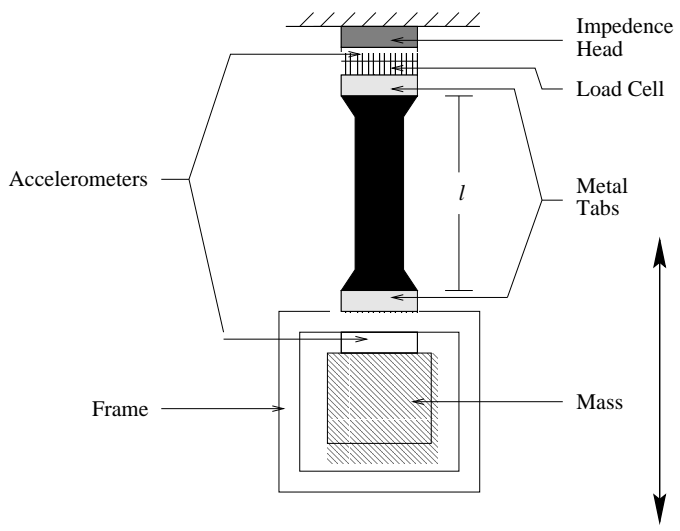

Figure 1: Rod with tip mass under tension.

infinitesimal strains $\varepsilon$ ) can be equivalently formulated in terms of nonlinear relationships between the stress and the infinitesimal strains $\varepsilon$. Hence we equivalently assume

$$
\sigma(t)=g_{e}\left(\frac{\partial u}{\partial x}\right)
$$

instead of a similar law involving the finite strains $\tilde{e}$.

The well-posedness of the initial boundary value problem associated with equations (2.1)-(2.4) was shown by Banks, Gilliam, Shubov in [3], while the convergence properties of the corresponding parameter estimation problem were established by Banks, Pinter in [7]. These results are valid for a broad class of nonlinearities. This is important since comparison between experimental data and numerical calculations suggested that the neo-Hookean nonlinearity (found in literature as a first approximation to the nonlinearity exhibited by rubber materials) is not adequate to describe the behavior of filled elastomers [6].

An adequate form of $g_{e}$ and the unknown constants $\rho$ and $C_{D}$ were determined using parameter estimation techniques. Data for the inverse problem were provided by dynamic free release experiments. The elastomer was suspended vertically with the top end $(x=0)$ fixed, and a frame was attached to the lower end (see Figure 1). Varying amounts of extra mass were attached to this frame, which also served to house an accelerometer. Another accelerometer, placed at the top of the sample, was used to verify the clamped boundary condition at the fixed end. For the free release experiment, the rubber rod was lifted together with the frame and the tip mass so that no compression or extension occurred. Then the support was removed, allowing the mass to fall freely. This type of experiment was repeated with unfilled and lightly filled carbon black samples, while a similar experiment was done with a highly filled sample with a $9.29 \mathrm{lb}$ tip mass.

The force data collected by the load cell on top of the sample were used in estimating the unknown parameters $q=\left\{g_{e}, \rho, C_{D}\right\}$ in (2.1)-(2.4) with (2.6), by minimizing

$$
J(q)=\frac{1}{2} \sum_{i=1}^{N}\left|z_{i}-A_{c} \sigma\left(\frac{\partial u}{\partial x}\left(t_{i}, 0 ; q\right)\right)\right|^{2}
$$

over $q$ in some admissible parameter space $Q$. Here $z_{i}, i=1, \ldots, N$ represent the experimental observations of the force at the fixed end, and $u$ is the solution of (2.1)-(2.4) corresponding to the parameters $q$. 
Computational results indicated that (1) a nonlinear function $g_{e}$ is necessary in the stressstrain relationship, (2) the stress-strain relationship (2.6) is adequate to describe unfilled rubber samples (Figure 2) and (3) to capture the dynamics of filled elastomers hysteresis must be taken into account.
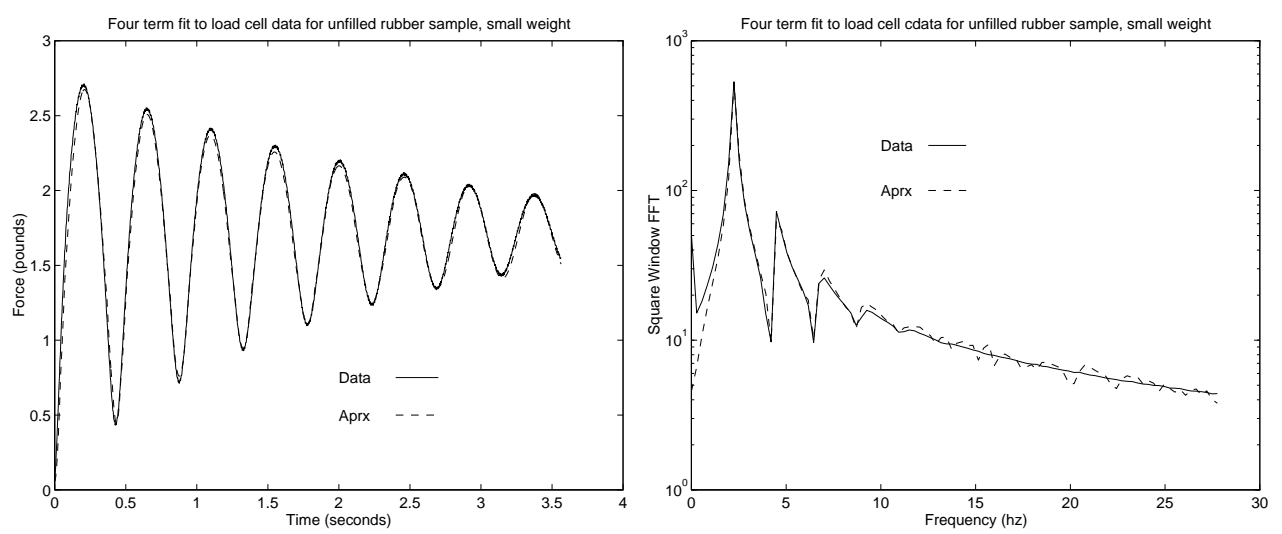

Figure 2: (left) Time domain approximation with a four-term piecewise linear $\tilde{g}$, and (right) the FFT of the solution and the data.

Based on models for hysteretic damping in the literature (see [8] and references therein) we assumed a Boltzmann-type stress-strain law of the form

$$
\sigma(t)=g_{e}(\varepsilon(t))+\int_{-\infty}^{t} Y(t-s) \frac{d}{d s} g_{v}(\varepsilon(s), \dot{\varepsilon}(s)) d s,
$$

where $Y$ is the memory kernel, and $g_{e}$ and $g_{v}$ are nonlinear functions accounting for the elastic and viscoelastic nonlinear responses of the elastomer, respectively. This stress-strain law implies that the stress depends not only on the current strain but also on the history of the strain and the strain-rate. It is very important to note that the stress-strain law (2.7) contains various standard internal strain or internal variable formulations as special cases. The ADF models of Lesieutre [19], [20] for composite materials exhibiting both elastic and anelastic displacement fields are formulated on the assumption that the host elastic material contains anelastic materials with internal strains $\varepsilon_{1}$ which are elastic strain driven. That is, the constitutive laws have the form

$$
\sigma(t)=E_{1} \varepsilon(t)-E_{2} \varepsilon_{1}(t)
$$

where the internal strain is given by

$$
\begin{aligned}
& \dot{\varepsilon}_{1}(t)+c_{1} \varepsilon_{1}(t)=c_{2} \varepsilon(t) \\
& \varepsilon_{1}(0)=0,
\end{aligned}
$$

or equivalently,

$$
\varepsilon_{1}(t)=\int_{0}^{t} c_{2} e^{-c_{1}(t-s)} \varepsilon(s) d s .
$$

Several generalizations of this formulation exist, e.g., Johnson et.al. [18], suggest that the internal strain is strain rate driven, i.e.,

$$
\dot{\varepsilon}_{1}(t)+c_{1} \varepsilon_{1}(t)=c_{2} \dot{\varepsilon}(t)
$$


Our Boltzmann-type law (2.7) (under appropriate assumptions on the past memory from $-\infty$ to 0 ) corresponds to an internal strain model of the form:

$$
\begin{aligned}
& \dot{\varepsilon}_{1}(t)+c_{1} \varepsilon_{1}(t)=\frac{d}{d t} g_{v}(\varepsilon(t), \dot{\varepsilon}(t)) \\
& \varepsilon_{1}(0)=0
\end{aligned}
$$

This form was chosen after we found that neither (2.9) nor (2.10) provided laws that could describe our data.

Experimental observations of the quasi-static behavior of elastomers indicate that these materials possess different nonlinear viscoelastic responses in loading $(\dot{\varepsilon}>0)$ and unloading $(\dot{\varepsilon}<0)$. This led to our choice of a piecewise continuous form for the viscoelastic response function $g_{v}$ :

$$
g_{v}(\varepsilon(s), \dot{\varepsilon}(s))= \begin{cases}g_{v i}(\varepsilon(s)) & \dot{\varepsilon}(s)>0 \\ g_{v d}(\varepsilon(s)) & \dot{\varepsilon}(s)<0 .\end{cases}
$$

Here we require $g_{v i}$ and $g_{v d}$ to be continuous (and generally nonlinear) functions. This difference between loading and unloading is more pronounced as the amount of filler increases in the material. We define the points $t_{i}, i=1, \ldots, M$ as the "turning points," or the points in time for which $\dot{\varepsilon}=0$. The function $g_{v}$ need not be continuous at the turning points, so we must interpret the derivatives in $(2.7),(2.11)$ as distributional derivatives. That is, delta functions are involved in differentiating the composite functions $g_{v}(\varepsilon, \dot{\varepsilon})$, or equivalently, integration by parts is valid. For experimental and computational purposes, we further assume that any motion in the material prior to each experiment is negligible. That is, we assume that $\frac{d}{d t} g_{v}(\varepsilon(t), \dot{\varepsilon}(t)) d t \approx 0$ for all $t<0$. Hence we may approximate (2.11) by

$$
\sigma(t)=g_{e}(\varepsilon(t))+\int_{0}^{t} Y(t-s) \frac{d}{d s} g_{v}(\varepsilon(s), \dot{\varepsilon}(s)) d s .
$$

We integrated by parts in (2.13) and obtained the model in variational form

$$
\begin{aligned}
& \tilde{\rho} \ddot{u}-C_{D} u_{x x t}-\frac{\partial}{\partial x}\left(A_{c} g_{e}\left(\frac{\partial u}{\partial x}\right)+A_{c} Y(0) g_{v}\left(\frac{\partial u}{\partial x}, \frac{\partial^{2} u}{\partial x \partial t}\right)+A_{c} \int_{0}^{t} \dot{Y}(t-s) g_{v}\left(\frac{\partial u}{\partial x}(s), \frac{\partial^{2} u}{\partial t \partial x}(s)\right) d s\right. \\
& \left.+A_{c} \sum_{k=1}^{K} Y\left(t-t_{k}\right)(-1)^{k+1}\left[g_{v i}\left(\frac{\partial u}{\partial x}\left(t_{k}\right)\right)-g_{v d}\left(\frac{\partial u}{\partial x}\left(t_{k}\right)\right)\right]\right)=F(t) \quad \text { in } V^{*} \\
& u(t, 0)=0, \quad u(0, x)=\varphi_{0}, \quad u_{t}(0, x)=0
\end{aligned}
$$

for an appropriately chosen Hilbert space $V$. This presumes, of course, that we have sufficient smoothness so that evaluation of $\frac{\partial u}{\partial x}$ at $t_{i}$ makes sense and $\frac{\partial}{\partial x}\left(g_{v i}\left(\frac{\partial u}{\partial x}\left(t_{i}\right)\right)\right), \frac{\partial}{\partial x}\left(g_{v d}\left(\frac{\partial u}{\partial x}\left(t_{i}\right)\right)\right) \in V^{*}$. For this particular example, one takes $V=H_{L}^{1}(0, \ell)=\left\{\phi \in L^{2}(0, \ell) \mid \phi^{\prime} \in L^{2}(0, \ell), \phi(0)=0\right\}$ and $\tilde{\rho}=\rho A_{c}-M \delta_{\ell}, \quad F(t)=-f(t) \delta_{\ell}$, where $\delta_{\ell}(x)$ is the Dirac function with atom at $x=\ell$. The important question of well-posedness of the system (2.14)-(2.15) is treated carefully in [9]. We have shown that under certain assumptions there exists a unique weak solution of the system (2.14)-(2.15). This result uses the Minty-Browder monotonicity method to treat the nonlinearities in the system and is valid for a broad range of elastic and viscoelastic response functions. 


\subsection{Experimental and computational results}

We first tested this model in the quasi-static behavior of filled rubber samples in uniaxial tension. Two different types of quasi-static pull tests were conducted. The first type (Type I) includes a sequence of loading and unloading the sample to produce load-displacement curves with decreasing maximum strain levels. In the second type of experiment (Type II), we created a sequence of strain loops that have decreasing maximum strain levels as before, but instead of a fixed minimum strain level we used progressively increasing minimum strain levels. (Full description of the quasi-static and dynamic tests and results are given in our paper [10].) We tried a number of linear and nonlinear functions for $g_{e}$ and $g_{v}$, including the special cases of $g_{e}$ and $g_{v}$ linear with $g_{v} \equiv g_{v i} \equiv g_{v d}$. The relative errors discussed in [25] suggested that nonlinear functions are necessary for both $g_{e}$ and $g_{v}$. Additional curve fitting studies outlined in [25] led to our choice of cubic polynomials for $g_{e}, g_{v i}$ and $g_{v d}$. That is, we chose parameterized nonlinearities of the form $g_{e}(x)=\sum_{i=1}^{3} E_{i} x^{i}, g_{v i}(x)=$ $\sum_{i=1}^{3} a_{i} x^{i}, \quad g_{v d}(x)=\sum_{i=1}^{3} b_{i} x^{i}$, where $E_{i}, a_{i}, b_{i}, i=1,2,3$ are real constants. Note that these response functions include no constant terms. Here we require $g_{e}(0)=g_{v i}(0)=g_{v d}(0)=0$ so that a zero strain will yield a zero stress according to our stress-strain relation. Based on additional studies detailed in [25], we chose an exponential form $Y(s)=e^{-C_{1} s}$ for the memory kernel. Such an exponential form generates totally nested hysteresis loops in the stress-strain curves, a feature also observed in our data.

The parameters $C_{1}, E_{i}, a_{i}$ and $b_{i}$ were estimated by setting up a least squares minimization problem using one or two of the outer stress and strain data loops from the experiments. Figure 3 shows results for a highly filled sample in Type I and Type II experiments, respectively. While the inverse problem was 'trained' on the largest loop only in Type I, and the two outer loops in the Type II experiment, we can see that there is a very good agreement between the data and the model predicted inside loops.
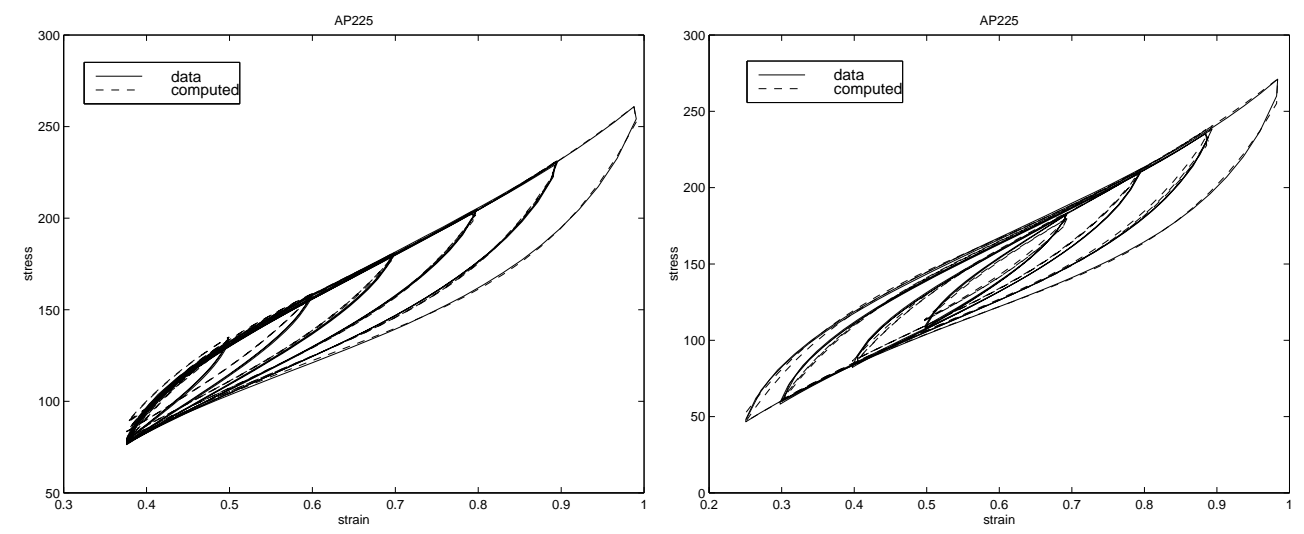

Figure 3: (left) Model prediction for Type I CB-h data and (right)Model prediction for Type II CB-h data .

Similar results were obtained for medium-filled natural rubber and silica-filled silicon samples. More details are given in [10].

Our hysteretic model (2.14)-(2.15) was also tested on a series of dynamical experiments with different types of filled rubber samples. We used the same free release experiments that were described earlier. Since the dynamical behavior of the unfilled natural rubber sample was adequately described by the basic model without hysteresis, we began our hysteresis investigations using the 
lightly filled sample. For given $\rho, g_{e}, g_{v}$ and $Y$ we solve the partial differential equation (2.14)(2.15) forward in time, and obtain the displacement $u(t, x), 0 \leq x \leq \ell$. The data collected in these experiments provides us with the force at the top of the sample $(x=0)$, and we compare it to the force predicted by the model at the same point, given by

$$
A_{c} \sigma(t, 0)=\left[C_{D} \dot{u}_{x}(t, 0)+A_{c} g_{e}\left(u_{x}(t, 0)\right)+A_{c} \int_{0}^{t} Y(t-s) \frac{d}{d s} g_{v}\left(u_{x}(s, 0), \dot{u}_{x}(s, 0)\right) d s\right],
$$

where we use our computed solution $u(t, x)$ to find $u_{x}(t, 0)$ and $\dot{u}_{x}(s, 0)$. Our goal is to find $\rho, g_{e}, g_{v}$ and $Y$ so that the model predicted force at $x=0$ best matches the data collected by the load cell. A parameter identification problem was set up to find $\rho, E_{1}, E_{2}, E_{3}, a_{1}, a_{2}, a_{3}, b_{1}, b_{2}, b_{3}, C_{1}$ (collectively denoted by $q$ ) such that

$$
J(q)=\sum_{i=1}^{M}\left|z_{i}-A_{c} \sigma\left(t_{i}, 0 ; q\right)\right|^{2}
$$

is minimized. Here the $z_{i}, i=1, \ldots, M$ are the data collected by the load cell, and $\sigma\left(t_{i}, 0 ; q\right)$ is given by the model. The particular forms for $g_{e}, g_{v}$ and $Y$ were motivated by their success in the quasi-static case. In our computations we used linear splines for spatial discretization. In solving the system (2.14)-(2.15) forward in time, the treatment of the hysteresis integral proved to be very time consuming. Since this computation needs to be repeated for each set of parameters during the optimization, the time required for the computational parameter identification process was very extensive. Hence, we formulated an equivalent system to (2.14)-(2.15) (with $\varphi_{0}, \varphi_{1}=0$ ) using internal variables and used it in the above framework for our subsequent calculations. This system is given by

$$
\begin{aligned}
& \tilde{\rho} \ddot{u}-C_{D} u_{x x t}-\frac{\partial}{\partial x}\left(A_{c} g_{e}\left(u_{x}\right)+A_{c} \varepsilon_{1}\right)=F(t) \quad \text { in } V^{*} \\
& \dot{\varepsilon}_{1}=-C_{1} \varepsilon_{1}+\frac{d}{d t}\left(g_{v}\left(u_{x}, \dot{u}_{x}\right)\right) \\
& u(t, 0)=0, \quad u(0, x)=u_{t}(0, x)=0, \quad \varepsilon_{1}(0, x)=0 \\
& u(t, x)=0, \quad t<0
\end{aligned}
$$

where, in general, the derivatives of $g_{v}$ in (2.17) are distributional in the sense described earlier. The parameter identification problem was solved using MATLAB optimization routines. Our results for the identification problem are shown in Figure 4, where a $3 \mathrm{lb}$ tip mass was used at the bottom of the sample.

The identification problem was also run on data obtained from experiments with $2 \mathrm{lb}$ and $1 \mathrm{lb}$ tip mass added to the lightly filled sample. We found a similar good agreement between data and the model [10]. However, the identified coefficients were not entirely consistent across experiments, although they should describe the same material. This variation is probably caused by the considerably different strain and strain rate ranges involved in the experiments, and suggests that the model should be refined to account for these differences.

We repeated the experiment with a highly filled sample having a $9.29 \mathrm{lb}$ tip mass. In this case our best fit depicted in Figure 5 (left) has deteriorated. Thus we turned to a modification of our model to obtain a better approximation to the force data.

Motivated by similar problems involving hysteretic effects and internal strain models we next tried to approximate the load cell data using two internal variables, $\varepsilon_{1}, \varepsilon_{2}$. Thus, we considered the model: 

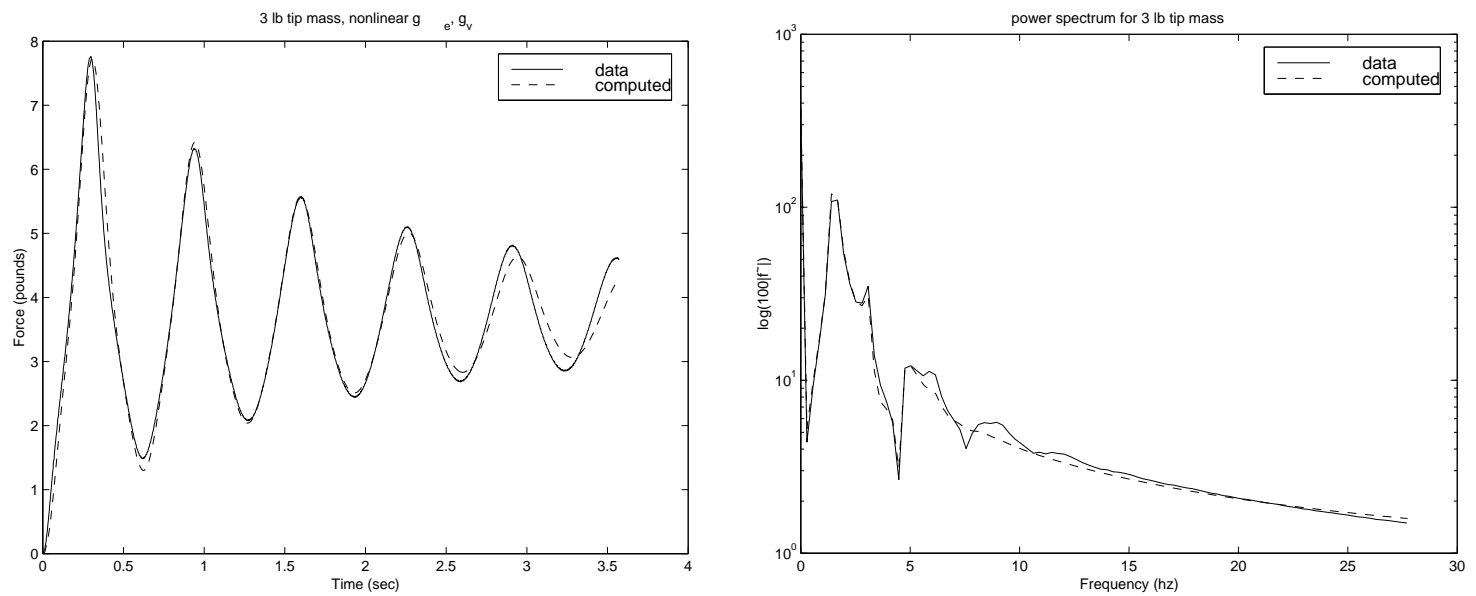

Figure 4: (left) Time domain approximation with a nonlinear $g_{e}, g_{v}, 3 \mathrm{lb}$ tip mass, $4.4 \%$ relative error, and (right) the power spectrum of the solution and the data.
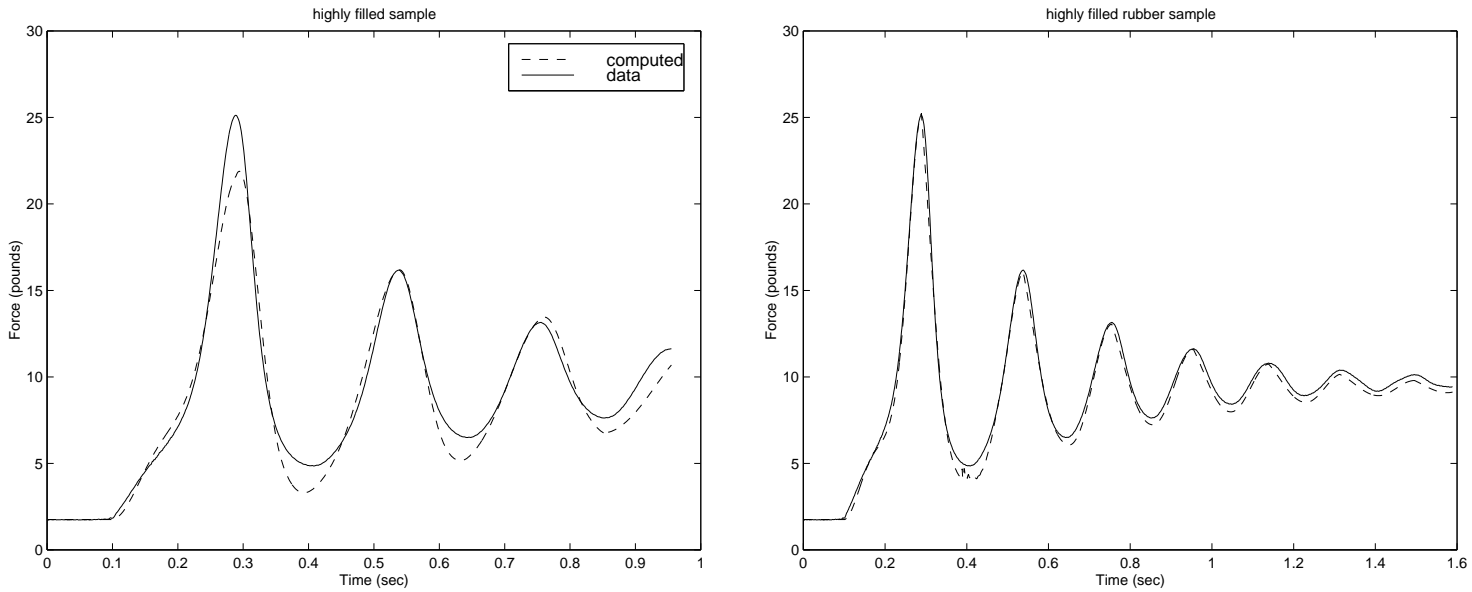

Figure 5: (left) Best approximation for the highly filled sample with one internal variable using data in the time interval [0,0.95] and (right)Best approximation for the highly filled sample with two internal variables on the full time interval. Identification based on data from the time interval $[0,0.95]$. 

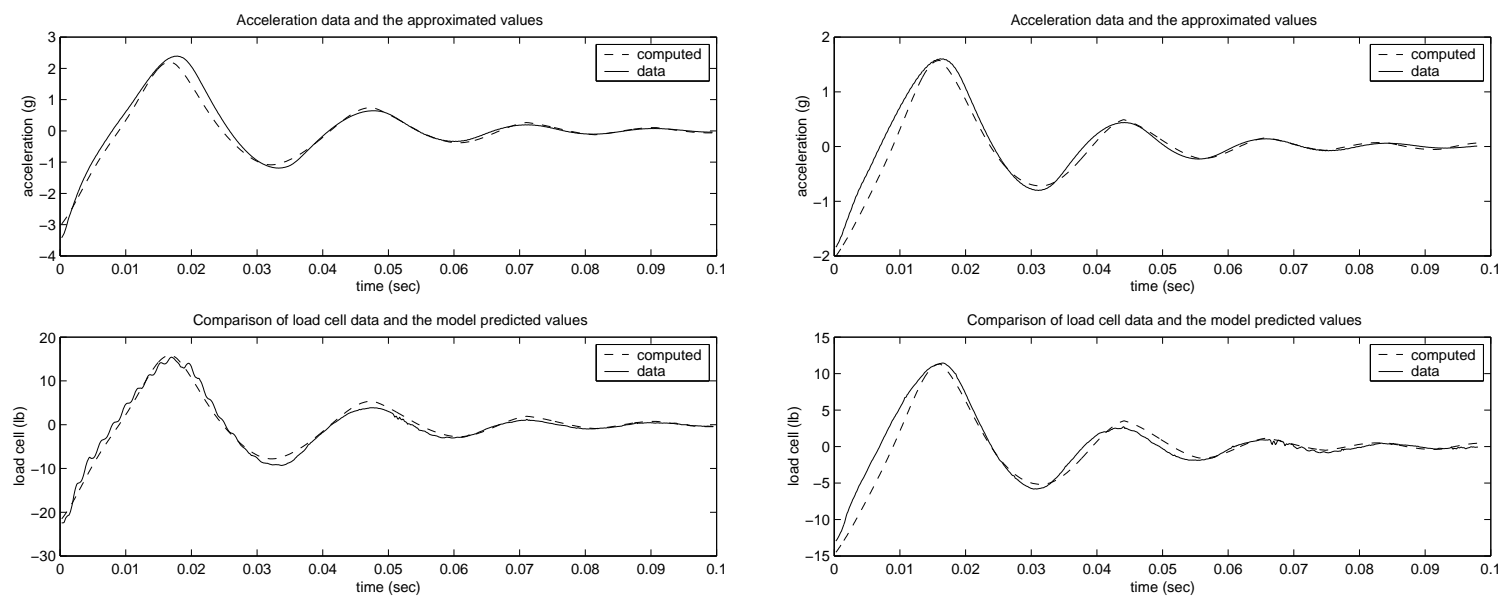

Figure 6: (left) Approximation result for the highly filled sample in shear with $\approx 100 \%$ strain initial condition and (right) The same parameter set simulating the highly filled sample in shear with $\approx$ $70 \%$ strain initial condition.

$$
\begin{aligned}
& \tilde{\rho} \ddot{u}-C_{D} u_{x x t}-\frac{\partial}{\partial x}\left(A_{c} g_{e}\left(u_{x}\right)+A_{c} \varepsilon_{1}+A_{c} \varepsilon_{2}\right)=F(t) \text { in } V^{*} \\
& \dot{\varepsilon}_{1}=-C_{1} \varepsilon_{1}+\frac{d}{d t}\left(g_{v}\left(u_{x}, \dot{u}_{x}\right)\right) \\
& \dot{\varepsilon}_{2}=-C_{2} \varepsilon_{1}+\frac{d}{d t}\left(g_{v}\left(u_{x}, \dot{u}_{x}\right)\right) \\
& u(t, 0)=0, \quad u(0, x)=\varphi_{0}, \quad u_{t}(0, x)=0 \\
& \varepsilon_{1}(0, x)=0, \quad \varepsilon_{2}(0, x)=0 .
\end{aligned}
$$

The parameter identification process gave very satisfactory results as shown in Figure 5 (right). The relative error between the data and the computed force is $4.1 \%$. We remark that this approximation was obtained by using data only from the time interval $[0,0.95]$, and resulted in a model that accurately simulated the data on the interval $[0,1.6]$.

We further note that the previous model development and identification process was repeated for highly filled elastomers in shear. Special experiments were designed and carried out at Lord Corporation to test this highly hysteretic rubber material in simple shear. Our model included a nonlinear elastic response function and nonlinear viscoelastic response functions through a Boltzmann integral term (in an internal variable formulation as in (2.16)-(2.17)). Results of the parameter estimation problem are depicted on Figure 6. The same parameter set describes two runs of the same experiment with different initial loading conditions with very good accuracy.

\subsection{Nonlinear internal variable models}

Our last result for the highly filled sample and the variation in the parameters for the lightly filled rubber rod suggested that we might try to generalize our model to better describe the behavior of highly hysteretic samples. Thus we next considered internal variable models with nonlinear internal 
dynamics:

$$
\begin{aligned}
& \tilde{\rho} \ddot{u}-C_{D} u_{x x t}-\frac{\partial}{\partial x}\left(A_{c} g_{e}\left(u_{x}\right)+A_{c} \varepsilon_{1}\right)=F(t) \text { in } V^{*} \\
& \dot{\varepsilon}_{1}=-g^{\varepsilon}\left(\varepsilon_{1}\right)+\frac{d}{d t}\left(g_{v}\left(u_{x}, \dot{u}_{x}\right)\right) \\
& u(t, 0)=0, \quad u(0, x)=\varphi_{0}, \quad u_{t}(0, x)=0 \\
& \varepsilon_{1}(0, x)=0 .
\end{aligned}
$$

In [1] we showed that this system has a unique weak solution. We also note that we in fact generalized the previous existence-uniqueness result in the sense that we no longer require monotonicity of the nonlinear functions $g_{e}, g_{v i}, g_{v d}$. Instead, they are assumed to satisfy a local Lipschitz property and other assumptions similar to those used before. We remark that similar techniques were successfully employed to establish existence-uniqueness of weak solutions for linear evolution equations of second order in $t$ in [13] and for semilinear second order evolution equations where the nonlinear forcing term satisfies a global Lipschitz condition in [17]. In [2] we used similar techniques to study a nonlinear beam equation where the nonlinearity satisfies only a local Lipschitz condition.

\section{Concluding Remarks}

In the above note we outlined our progress to date in the development of nonlinear dynamic models for inactive filled elastomers. Substantial experimental validation for our approach is provided both in the quasi-static and dynamic cases in uniaxial tension and in the dynamic case in simple shear.Current efforts involve refinements to these models and a comparison with newly developed molecular based models [5].

\section{Acknowledgements}

This research was supported in part by the U.S. Airforce Office of Scientific Research under grants AFOSR F49620-98-1-0180 (HTB and GAP) and AFOSR F49620-98-1-0430 (LKP).

\section{References}

[1] A.C. Ackleh, H.T. Banks, G.A. Pinter, Well-posedness results for models of elastomers, CRSC Tecnical Report, CRSC-TR00-21, September, 2000; J. Math. Analysis and Applications to appear.

[2] A.C. Ackleh, H.T. Banks, G.A. Pinter, On a nonlinear beam equation, CRSC Technical Report, CRSC-TR00-14, May, 2000; Applied Math. Letters, to appear.

[3] H.T. Banks, D.S. Gilliam and V.I. Shubov, Glob D.S. Gilliam and V.I. Shubov, Global solvability for damped abstract nonlinear hyperbolic systems, Differential and Integral Equations, 10, 1997, 309-332.

[4] H.T. Banks and N. Lybeck, Modeling methodology for elastomer dynamics, in Systems and Control in the 21st Century, Birkhäuser, Boston, 1996, 37-50. 
[5] H.T. Banks and N.G. Medhin, A molecular based dynamic model for viscoelastic pesponses of rubber in tensile deformations, NCSU Technical Report, CRSC-TR00-27, October, 2000.

[6] H.T. Banks, N.J. Lybeck, M.J. Gaitens, B.C. Muñoz and L.C. Yanyo, Computational methods for estimation in the modeling of nonlinear elastomers, CRSC-TR95-40, NCSU; Kybernetika, 32, 1996, 526-542.

[7] H.T. Banks and G.A. Pinter, Approximation results for parameter estimation in nonlinear elastomers, CRSC Technical Report, CRSC-TR96-34, December, 1996; Control and Estimation of Distributed Parameter Systems, F. Kappel, et.al.,eds., Birkhäuser, Boston, 1997.

[8] H.T. Banks and G.A. Pinter, Damping: hysteretic damping an Damping: hysteretic damping and models, CRSC Technical Report, CRSC-TR99-36, December, 1999; Encyclopedia of Vibrations to appear.

[9] H.T. Banks, G.A. Pintér, and L.K. Potter, Existence of unique weak solutions to a dynamical system for nonlinear elastomers with hysteresis, CRSC Technical Report CRSC-TR9843, Nov. 1998; Differential and Integral Equations, 13, 2000, 1001-1024.

[10] H.T. Banks, G.A. Pintér, and L.K. Potter, Modeling of nonlinear hysteresis in elastomers under uniaxial tension, J. Intelligent Mat. Systems and Structures, 10, 1999, 116-134.

[11] H.T. Banks, R.C. Smith and Y. Wang, Smart Material Structures: Modeling, Estimation and Control, Masson, Paris and John Wiley and Sons, Chichester, 1996.

[12] R.M. Christensen, Theory of Viscoelasticity: An Introduction, 2nd ed., Academic Press, New York, 1982.

[13] R. Dautray and J.L. Lions, Mathematical Analysis and Numerical Methods for Science and Technology, Volume 5, Evolution Problems I, Springer, New York, 2000.

[14] W.N. Findley, J.S. Lai and K. Onaran, Creep and Relaxation of Nonlinear Viscoelastic Materials, H.A. Lauwerier and W.T. Koiter, eds., North-Holland Series in Applied Mathematics and Mechanics, North-Holland Publishing Company, 1976.

[15] J.D. Ferry, Viscoelastic Properties of Polymers, John Wiley \& Sons, Inc., New York, 1980.

[16] M.V. Gandhi and B.S. Thompson, Smart Materials and Structures, Chapman and Hall, London, 1992.

[17] J. Ha and S. Nakagiri, Existence and regularity of weak solutions for semilinear second order evolution equations, Funkcialaj Ekvacioj, 41, 1998, 1-24.

[18] A.R. Johnson, C.J. Quigley and J.L. Mead, Large strain viscoelastic constitutive models for rubber, part I: Formulations, Rubber Chemistry Technology, 67, 1994, 904-917.

[19] G.A. Lesieutre, Modeling frequency-dependent longitudinal dynamic behavior of linear viscoelastic long fiber components, J. Composite Materials, 28, 1994, 1770-1782. 
[20] G.A. Lesieutre and K. Govindswamy, Finite element modeling of frequency-dependent and temperature-dependent dynamic behavior of viscoelastic materials in simple shear, Int. J. Solids Structures, 33, 1996, 419-432.

[21] R.W. Ogden, Non-linear Elastic Deformations, Ellis Horwood Limited, Chichester, 1984.

[22] R.S. Rivlin, Large elastic deformations of isotropic materials, I, II, III. Phil. Trans. Roy. Soc. A, 240, 1948, 459-525.

[23] R.A. Schapery, On the characterization of nonlinear viscoelastic materials, Polymer Engineering and Science, 9, 1969, 295-310.

[24] I.M. Ward, Mechanical Properties of Solid Polymers, John Wiley \& Sons, New York, 2nd ed., 1983.

[25] Y. Zhang, Mathematical formulation of vibrations of a composite curved beam structure: Aluminum core material with viscoelastic layers, constraining layers and piezoceramic patches, Ph.D. Thesis, N.C. State University, May 1997. 